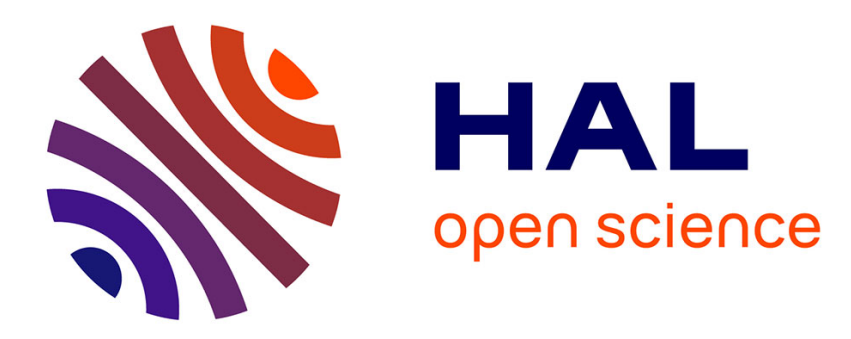

\title{
Edge-Face Coloring of Plane Graphs with Maximum Degree Nine
}

\author{
Jean-Sébastien Sereni, Matej Stehlík
}

\section{To cite this version:}

Jean-Sébastien Sereni, Matej Stehlík. Edge-Face Coloring of Plane Graphs with Maximum Degree Nine. Journal of Graph Theory, 2011, 66 (4), pp.332-346. 10.1002/jgt.20506 . hal-00574164

\section{HAL Id: hal-00574164 \\ https://hal.science/hal-00574164}

Submitted on 7 Mar 2011

HAL is a multi-disciplinary open access archive for the deposit and dissemination of scientific research documents, whether they are published or not. The documents may come from teaching and research institutions in France or abroad, or from public or private research centers.
L'archive ouverte pluridisciplinaire HAL, est destinée au dépôt et à la diffusion de documents scientifiques de niveau recherche, publiés ou non, émanant des établissements d'enseignement et de recherche français ou étrangers, des laboratoires publics ou privés. 


\title{
Edge-face colouring of plane graphs with maximum degree nine
}

\author{
Jean-Sébastien Sereni* Matěj Stehlík ${ }^{\dagger}$
}

\begin{abstract}
An edge-face colouring of a plane graph with edge set $E$ and face set $F$ is a colouring of the elements of $E \cup F$ so that adjacent or incident elements receive different colours. Borodin [Simultaneous coloring of edges and faces of plane graphs, Discrete Math., 128(1-3):21-33, 1994] proved that every plane graph of maximum degree $\Delta \geqslant 10$ can be edge-face coloured with $\Delta+1$ colours. We extend Borodin's result to the case where $\Delta=9$.
\end{abstract}

\section{Introduction}

Let $G$ be a plane graph with vertex set $V$, edge set $E$ and face set $F$. Two faces of $G$ are adjacent if the corresponding vertices of the dual of $G$ are. An edge $e$ and a face $f$ of $G$ are incident if $e$ belongs to the boundary walk of $f$. Given a positive integer $k$, a $k$-edge-face colouring of $G$ is a mapping $\lambda: E \cup F \rightarrow\{1,2, \ldots, k\}$ such that

(i) $\lambda(e) \neq \lambda\left(e^{\prime}\right)$ for every pair $\left(e, e^{\prime}\right)$ of adjacent edges;

(ii) $\lambda(e) \neq \lambda(f)$ for edge $e$ and every face $f$ incident to $e$;

(iii) $\lambda(f) \neq \lambda\left(f^{\prime}\right)$ for every pair $\left(f, f^{\prime}\right)$ of adjacent faces with $f \neq f^{\prime}$.

The requirement in (iii) that $f$ and $f^{\prime}$ be distinct is only relevant for graphs containing a cut-edge; such graphs would not have an edge-face colouring otherwise.

${ }^{*}$ CNRS (LIAFA, Université Denis Diderot), Paris, France and Department of Applied Mathematics (KAM), Faculty of Mathematics and Physics, Charles University, Prague, Czech Republic (sereni@kam.mff.cuni.cz).

${ }^{\dagger}$ LIAFA, Université Denis Diderot (Paris 7), Paris, France (matej@liafa.jussieu.fr). 
Edge-face colourings appear to have been first studied by Jucovič 8 and Fiamčík [7], who considered 3- and 4-regular graphs. Mel'nikov [1] conjectured that every plane graph of maximum degree $\Delta$ has a $(\Delta+3)$-edgeface colouring. This was proved by Borodin [2, 4] for $\Delta \leqslant 3$ and $\Delta \geqslant 8$, and the general case was proved by Waller [15], and independently by Sanders and Zhao [12]. In fact, Borodin [4] proved the upper bound of $\Delta+1$ for plane graphs of maximum degree $\Delta \geqslant 10$. The bound is tight, as can be seen by considering trees. Borodin asked [4, Problem 9] to determine the exact upper bound for plane graphs with maximum degree $\Delta \leqslant 9$. We solve the problem for the case $\Delta=9$ by proving the following theorem.

Theorem 1. Every plane graph of maximum degree at most 9 has a 10-edgeface colouring.

Borodin's problem remains open $\prod^{1}$ for graphs of maximum degree $\Delta \in$ $\{4,5, \ldots, 8\}$. Note that Sanders and Zhao [13] have proved that plane graphs of maximum degree $\Delta \geqslant 7$ are $(\Delta+2)$-edge-face colourable.

Let us briefly mention the closely related concept of total colouring: given a graph $G=(V, E)$, we colour the elements of $V \cup E$ so that adjacent or incident elements receive different colours. The well-known Total Colouring Conjecture of Behzad [1] and Vizing [14] states that every graph of maximum degree $\Delta$ admits a $(\Delta+2)$-total-colouring. For planar graphs of maximum degree $\Delta \geqslant 14$, Borodin [3] proved the stronger bound $\Delta+1$. This bound was subsequently extended to graphs of maximum degree $\Delta \in\{9,10, \ldots, 13\}$ by Borodin, Kostochka and Woodall [5, 6], by Wang [16], and by Kowalik, Sereni and Škrekovski [10].

We prove Theorem 1 by contradiction. From now on, we let $G=(V, E, F)$ be a counter-example to the statement of Theorem 1 with as few edges as possible. That is, $G$ is a plane graph of maximum degree 9 that admits no 10-edge-face colouring, but every plane graph of maximum degree 9 with less than $|E|$ edges has a 10-edge-face colouring. In particular, for every edge $e \in E$ the plane subgraph $G-e$ of $G$ has a 10-edge-face colouring. First, we establish various structural properties of $G$ in Section 2. Then, relying on these properties, we use the Discharging Method in Section 3 to obtain a contradiction.

In the sequel, a vertex of degree $d$ is called a $d$-vertex. A vertex is an $(\leqslant d)$ vertex if its degree is at most $d$; it is an $(\geqslant d)$-vertex if its degree is at least $d$. The notions of $d$-face, $(\leqslant d)$-face and $(\geqslant d)$-face are defined analogously as for the vertices, where the degree of a face is the length of its boundary walk.

\footnotetext{
${ }^{1}$ In a forthcoming paper 9 , Ross Kang and the authors prove that every plane graph of maximum degree at most 8 has a 9-edge-face colouring, thereby solving the case $\Delta=8$.
} 
A face of length 3 is called a triangle. For integers $a, b, c$, an $(\leqslant a, \leqslant b, \leqslant c)$ triangle is a triangle $x y z$ of $G$ with $\operatorname{deg}(x) \leqslant a, \operatorname{deg}(y) \leqslant b$ and $\operatorname{deg}(z) \leqslant c$. The notions of $(a, \leqslant b, \leqslant c)$-triangles, $(a, b, \geqslant c)$-triangles and so on, are defined analogously.

\section{Reducible configurations}

In this section, we establish some structural properties of the graph $G$. In particular, we prove that some plane graphs are reducible configurations, i.e. they cannot be part of the chosen embedding of $G$.

For convenience, we sometimes define configurations by depicting them in figures. We use the following conventions: 2- and 3-vertices are depicted by small black bullets and black triangles, respectively; vertices of degree at most 5 are represented by black pentagons, and white bullets represent vertices of degree at least as large as the one shown in the figure (and made precise in the text, if necessary). Furthermore, the colour of a face is shown in a box on that face, to avoid confusion with the colours of the edges.

Let $\lambda$ be a (partial) 10-edge-face colouring of $G$. For each element $x \in$ $E \cup F$, we define $\mathcal{C}(x)$ to be the set of colours (with respect to $\lambda$ ) of the edges and faces incident or adjacent to $x$. Also, we set $\mathcal{F}(x):=\{1,2, \ldots, 10\} \backslash \mathcal{C}(x)$. If $x \in V$ we define $\mathcal{E}(x)$ to be the set of colours of the edges incident to $x$. Moreover, $\lambda$ is nice if only some $(\leqslant 4)$-faces are uncoloured. Observe that every nice colouring can be greedily extended to a 10-edge-face colouring of $G$, since $|\mathcal{C}(f)| \leqslant 8$ for each $(\leqslant 4)$-face $f$, i.e. $f$ has at most eight forbidden colours. Therefore, in the rest of the paper, we shall always suppose that such faces are coloured at the very end. More precisely, every time we consider a partial colouring of $G$, we uncolour all $(\leqslant 4)$-faces, and implicitly colour them at the very end of the colouring procedure of $G$. We make the following observation about nice colourings.

Observation. Let $e$ be an edge incident to two faces $f$ and $f^{\prime}$. There exists a nice colouring $\lambda$ of $G-e$, and hence a partial 10-edge-face colouring of $G$ in which only $e$ and $f$ are uncoloured. Moreover, if $f$ is an $(\leqslant 4)$ face, then it suffices to properly colour the edge $e$ (i.e. with a colour from $\{1,2, \ldots, 10\} \backslash \mathcal{C}(e))$ to extend $\lambda$ to a nice colouring of $G$.

This observation is used throughout the paper.

Lemma 2. The graph $G$ has the following properties.

( $i$ ) Let $v$ be a vertex of $G$, and $v_{0}, v_{1}, \ldots, v_{d-1}$ its neighbours in clockwise order in the embedding of $G$. If $v$ is a cut-vertex of $G$, then no component 
$C$ of $G-v$ is such that the neighbourhood of $v$ in $C$ is contained in $\left\{v_{i}, v_{i+1}\right\}$ for some $i \in\{0,1, \ldots, d-1\}$, where the indices are taken modulo d. In particular, $G$ has no cut-edge.

(ii) If uv is an edge incident to a 5-face then $\operatorname{deg}(u)+\operatorname{deg}(v) \geqslant 10$.

(iii) Let uv be an edge, and let $x \in\{1,2\}$ be the number of $(\leqslant 4)$-faces incident to uv. Then $\operatorname{deg}(u)+\operatorname{deg}(v) \geqslant 10+x$.

Proof. $(i)$. Suppose on the contrary that $C$ is a component of $G-v$ such that the neighbourhood $N$ of $v$ in $C$ is contained in, say, $\left\{v_{0}, v_{1}\right\}$.

First, assume that $N=\left\{v_{0}, v_{1}\right\}$. Then $G$ is the edge-disjoint union of two plane graphs $G_{1}=\left(C \cup\{v\}, E_{1}\right)$ and $G_{2}=\left(V \backslash C, E_{2}\right)$. The outer face $f_{1}$ of $G_{1}$ corresponds to a face $f_{2}$ of $G_{2}$. By the minimality of $G$, the graph $G_{i}$ has a 10-edge-face colouring $\lambda_{i}$ for $i \in\{1,2\}$. Since both $v v_{0}$ and $v v_{1}$ are incident in $G_{1}$ to $f_{1}$, we may assume that $\lambda_{1}\left(f_{1}\right)=1, \lambda_{1}\left(v v_{0}\right)=9$ and $\lambda_{1}\left(v v_{1}\right)=10$. Regarding $\lambda_{2}$, we may assume that $\lambda_{2}\left(f_{2}\right)=1$. Furthermore, up to permuting the colours, we can also assume that the colours of the edges of $G_{2}$ incident to $v$ are contained in $\{1,2, \ldots, 8\}$, since there are at most seven such edges.

We now define an edge-face colouring $\lambda$ of $G$ as follows. For every edge $e$ of $G$, set $\lambda(e):=\lambda_{1}(e)$ if $e \in E_{1}$ and $\lambda(e):=\lambda_{2}(e)$ if $e \in E_{2}$. To colour the faces of $G$, let $f$ be the face of $G$ incident to both $v v_{0}$ and $v v_{d-1}$ (note that there is only one such face, since $v_{0}$ and $v_{d-1}$ are in distinct connected components of $G-v$ ). Now, observe that there is a natural one-to-one correspondence between the faces of $G_{1}$ and a subset $F_{1}$ of the face set $F$ of $G$ that maps $f_{1}$ to $f$. Similarly, there is a natural one-to-one correspondence between the faces of $G$ and a subset $F_{2}$ of $F$ that maps $f_{2}$ to $f$. Note that $F_{1} \cap F_{2}=\{f\}$. Now, we can colour every face $f \in F_{i}$ using $\lambda_{i}$. This is well defined since $\lambda_{1}\left(f_{1}\right)=\lambda_{2}\left(f_{2}\right)=1$.

Let us check that $\lambda$ is proper. Two adjacent edges of $G$ are assigned different colours. Indeed, if the two edges belong to $E_{i}$ for some $i \in\{1,2\}$, then it comes from the fact that $\lambda_{i}$ is a proper edge-face colouring of $G_{i}$. Otherwise, both edges are incident with $v$, and one is in $G_{1}$ and the other in $G_{2}$. The former is coloured either 9 or 10 , and the latter with a colour of $\{1,2, \ldots, 8\}$ by the choice of $\lambda_{1}$ and $\lambda_{2}$. Two adjacent faces in $G$ necessarily correspond to two adjacent faces in $G_{1}$ or $G_{2}$, and hence are assigned different colours. Last, let $g$ be a face of $G$ and $e$ an edge incident to $g$ in $G$. If $g \neq f$, then $g$ and $e$ are incident in $G_{1}$ or $G_{2}$, and hence coloured differently. Otherwise $e$ is incident to $f_{i}$ in $G_{i}$ for some $i \in\{1,2\}$, and hence $\lambda(e)=\lambda_{i}(e) \neq \lambda_{i}\left(f_{i}\right)=1=\lambda(f)$.

The case where $N=\left\{v_{0}\right\}$, i.e. $v v_{0}$ is a cut-edge, is dealt with in the very same way so we omit it. 
$(i i)$. Let $e=u v$ be an edge with $\operatorname{deg}(u)+\operatorname{deg}(v) \leqslant 9$ and let $f$ and $f^{\prime}$ be the two faces incident to $e$. Suppose that $f$ is a 5 -face. By the minimality of $G$, the graph $G-e$ has a nice colouring $\lambda$. Let $f^{\prime \prime}$ be the face of $G-e$ corresponding to the union of the two faces $f$ and $f^{\prime}$ of $G$ after having removed the edge $e$. We obtain a partial 10-edge-face colouring of $G$ in which only $e, f$ and the $(\leqslant 4)$-faces are uncoloured by just assigning the colour $\lambda\left(f^{\prime \prime}\right)$ to $f^{\prime}$, and keeping all the other assignments. Since $f$ is a 5 -face and $e$ is uncoloured, $|\mathcal{C}(f)| \leqslant 9$. Thus, we can properly colour $f$. Now, $|\mathcal{C}(e)| \leqslant \operatorname{deg}(u)+\operatorname{deg}(v)-2+2 \leqslant 9$. Hence, the edge $e$ can be properly coloured, which yields a nice colouring of $G$; a contradiction.

(iii). Suppose on the contrary that $\operatorname{deg}(u)+\operatorname{deg}(v) \leqslant 9+x$. Let $f$ and $f^{\prime}$ be the two faces incident to $u v$, with $f$ being an $(\leqslant 4)$-face. By the minimality of $G$, the graph $G-e$ has a nice colouring. We obtain a partial 10 -edge-face colouring of $G$ as above (in particular, only $e$ and the $(\leqslant 4)$-faces are uncoloured). Consequently, $|\mathcal{C}(u v)| \leqslant \operatorname{deg}(u)+\operatorname{deg}(v)-2+2-x \leqslant 9$. Hence, we can properly colour the edge $u v$, thereby obtaining a nice colouring of $G$; a contradiction.

Lemma 3. The graph $G$ satisfies the following assertions.

(i) Two vertices of degree 2 are not adjacent.

(ii) A triangle is not incident to a 2-vertex.

(iii) A 4-face incident to a 2-vertex is not incident to another ( $\leqslant 3)$-vertex.

Proof. ( $(i)$. Suppose on the contrary that $v_{1}$ and $v_{2}$ are two adjacent 2-vertices. For $i \in\{1,2\}$, let $u_{i}$ be the neighbour of $v_{i}$ other than $v_{3-i}$. Note that $u_{1} \neq u_{2}$ by Lemma 2( $(i)$. In particular $v_{1}$ and $u_{2}$ are not adjacent. Let $G^{\prime}$ be the plane graph obtained from $G$ by suppressing $v_{2}$, i.e. removing $v_{2}$ and adding an edge between $v_{1}$ and $u_{2}$. By the minimality of $G$, the graph $G^{\prime}$ has a 10-edge-face colouring $\lambda^{\prime}$. Observe that $\lambda^{\prime}$ naturally defines a partial 10-edge-face colouring $\lambda$ of $G$ in which only the edge $v_{1} v_{2}$ is uncoloured. Indeed, every face $f$ of $G$ naturally corresponds to a face $f^{\prime}$ of $G^{\prime}$, so setting $\lambda(f):=\lambda^{\prime}\left(f^{\prime}\right)$ yields a proper colouring of the faces of $G$. If $e$ is an edge not incident to $v_{2}$ then $e$ is also an edge of $G^{\prime}$, and we set $\lambda(e):=\lambda^{\prime}(e)$. Next, we colour $u_{2} v_{2}$ with $\lambda^{\prime}\left(u_{1} v_{2}\right)$. Now, $\left|\mathcal{C}\left(v_{1} v_{2}\right)\right|=\operatorname{deg}\left(v_{1}\right)+\operatorname{deg}\left(v_{2}\right)-2+2=4<10$, so we can greedily colour $v_{1} v_{2}$, thereby obtaining a 10-edge-face colouring of $G$.

(ii). Suppose that $f:=u v w$ is a 3 -face and $v$ a 2 -vertex. By Lemma2 2(iii), the vertices $u$ and $v$ both have degree 9 . By the minimality of $G$, the graph $G^{\prime}:=G-u v$ has a nice colouring. Thus, we obtain a partial 10-edge-face 
colouring of $G$ in which only $u v$ and the $(\leqslant 4)$-faces are uncoloured. It suffices to properly colour the edge $u v$ to obtain a nice colouring of $G$, and hence a contradiction. If we cannot do this greedily, then $|\mathcal{C}(u v)|=10$ so we can assume without loss of generality that the colouring is the one shown in Figure $1(a)$. Let $x \in\{1,2, \ldots, 7\}$. If $x \notin \mathcal{E}(w)$, then we can recolour the edge $v w$ with $x$, and colour $u v$ with 9 to obtain a nice colouring of $G$. Thus, $\mathcal{E}(w)=\{1,2, \ldots, 9\}$.

Now, let $g$ be the face incident to $u w$ other than $f$, so the colour of $g$ is $\alpha$. We assert that $\alpha \neq 10$. To see this, let $f^{\prime}$ be the face of $G^{\prime}$ corresponding to the union of the two faces incident to $u v$. Observe that in $G^{\prime}$, the faces $f^{\prime}$ and $g$ are not the same, for otherwise $u$ would be a cut-vertex that contradicts Lemma 2|(i). Therefore $f^{\prime}$ and $g$ are adjacent in $G^{\prime}$, and thus the assertion holds. Consequently, we can recolour $u w$ with 10 and colour $u v$ with 8 to obtain a nice colouring of $G$; a contradiction.

(iii). Suppose on the contrary that $v u u^{\prime} v^{\prime}$ is a 4 -face incident to a 2 -vertex $u$ and an $(\leqslant 3)$-vertex. By Lemma $2($ iii $)$, we may assume that $v^{\prime}$ is an $(\leqslant 3)$ vertex and $v$ and $u^{\prime}$ are 9 -vertices. By the minimality of $G$, the graph $G-u v$ has a nice colouring, from which we infer a partial 10-edge-face colouring of $G$ in which only the edge $u v$ and the $(\leqslant 4)$-faces are uncoloured. It suffices to properly colour the edge $u v$ to obtain a nice colouring of $G$, and hence a contradiction. If we cannot do this greedily, then $|\mathcal{C}(u v)|=10$ so we can assume without loss of generality that the colouring is of the form shown in either Figure $1(b)$ or $1(c)$.

Notice that $\{9,10\} \subset \mathcal{C}\left(v v^{\prime}\right)$, otherwise we can recolour $v v^{\prime}$ with 9 or 10 and then colour $u v$ with 1 . Moreover, $\delta \notin\{9,10\}$ as $u u^{\prime}$ is coloured with 9 and $u^{\prime} v^{\prime}$ is incident to a face coloured with 10 in $G-u v$. So if $v^{\prime}$ is a 2-vertex then $\{9,10\} \nsubseteq \mathcal{C}\left(v v^{\prime}\right)$; a contradiction.

Thus, we may now assume that $v^{\prime}$ is a 3 -vertex, and hence $\{9,10\}=\{\alpha, \beta\}$. Recalling that $G$ has no cut-edge by Lemma $2(i)$, we deduce that $\gamma \notin\{9,10\}$. Further, $\beta \neq 10$ for otherwise $v v^{\prime}$ would be a cut-edge of $G-u v$, and thus $v$ would be a cut-vertex of $G$ that contradicts Lemma 2| $(i)$. Hence, $\beta=9$ and $\alpha=10$. Now, colouring $u v$ with 9 and recolouring $u u^{\prime}$ with $\delta$ and $u^{\prime} v^{\prime}$ with 9 yields a nice colouring of $G$, and the desired contradiction.

Lemma 4. Let uvw be a 3 -face of $G$ such that $\operatorname{deg}(u)+\operatorname{deg}(v) \leqslant 11$. Then $\operatorname{deg}(w) \geqslant 8$. In particular, $G$ has no $(4,7,7)$-triangle and no $(5,6, \leqslant 7)$ triangle.

Proof. Suppose on the contrary that $\operatorname{deg}(w) \leqslant 7$, and assume without loss of generality that $\operatorname{deg}(u) \leqslant \operatorname{deg}(v)$. We obtain from a nice colouring of $G-u v$ a 


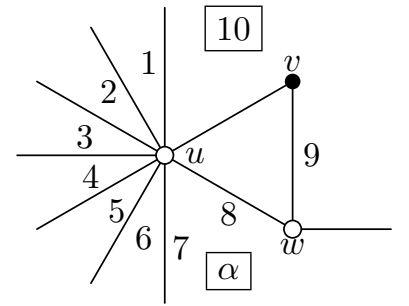

(a)

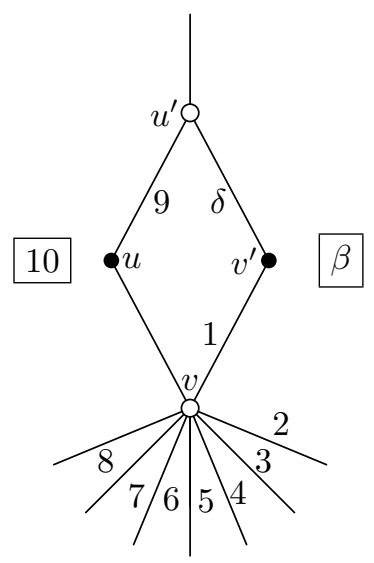

(b)

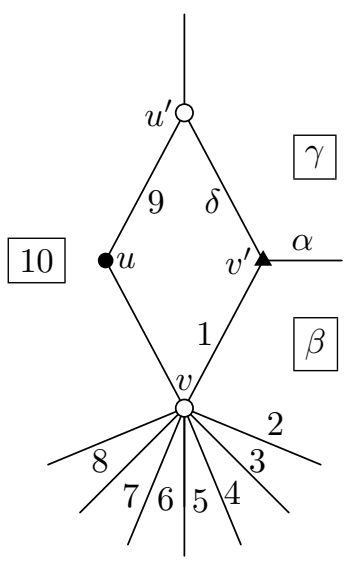

(c)

Figure 1: Reducible configurations for the proof of Lemma 3.

partial 10-edge-face colouring $\lambda$ of $G$ in which only $u v$ and the $(\leqslant 4)$-faces are uncoloured. To extend $\lambda$ to a nice colouring of $G$, it suffices to properly colour the edge $u v$. If we cannot do this greedily, it means that $|\mathcal{C}(u v)|=10$. Thus, since $|\mathcal{C}(u v)| \leqslant \operatorname{deg}(u)+\operatorname{deg}(v)-2+1=10$, we deduce that $\mathcal{E}(u) \cap \mathcal{E}(v)=\emptyset$.

We obtain a contradiction by using a counting argument. We assert that $\eta:=|\mathcal{E}(u) \cap \mathcal{E}(w)| \leqslant \operatorname{deg}(u)+\operatorname{deg}(w)-10$. To see this, observe that if $|\mathcal{C}(u w)| \leqslant 8$, then $u w$ can be properly recoloured with a colour different from $c:=\lambda(u w)$, and subsequently $u v$ can be coloured with $c$; a contradiction. But $|\mathcal{C}(u w)| \leqslant(\operatorname{deg}(u)-2)+(\operatorname{deg}(w)-1)-(\eta-1)+1$. So $\eta \leqslant \operatorname{deg}(u)+\operatorname{deg}(w)-10$, as asserted. Similarly, $\eta^{\prime}:=|\mathcal{E}(v) \cap \mathcal{E}(w)| \leqslant \operatorname{deg}(v)+\operatorname{deg}(w)-10$.

Now set $\nu:=|\mathcal{E}(u) \cup \mathcal{E}(v) \cup \mathcal{E}(w)|$. Since $\mathcal{E}(u) \cap \mathcal{E}(v)=\emptyset$, we obtain

$$
\begin{aligned}
\nu & =(\operatorname{deg}(u)-1)+(\operatorname{deg}(v)-1)+\operatorname{deg}(w)-\eta-\eta^{\prime} \\
& \geqslant 20-2-\operatorname{deg}(w) \\
& \geqslant 11
\end{aligned}
$$

a contradiction.

Lemma 5. Let $v$ be an 8-vertex of $G$, and $v_{0}, v_{1}, \ldots, v_{7}$ the neighbours of $v$ in anti-clockwise order in the embedding of $G$. For $i \in\{0,1, \ldots, 7\}$, let $f_{i}$ be the face of $G$ incident to $v v_{i}$ and to $v v_{i+1}$, where the indices are taken modulo 8. Assume that $f_{0}$ is a $(3,8, \geqslant 8)$-triangle.

(i) The face $f_{1}$ is not a $(3,8, \geqslant 8)$-triangle; and

(ii) if the face $f_{3}$ is a $(3,8, \geqslant 8)$-triangle and $f_{1}$ and $f_{2}$ are 3 -faces, then $\operatorname{deg}\left(v_{2}\right) \geqslant 6$. 
Proof. We proceed by contradiction in both cases, by assuming that $v$ contradicts the considered statement.

$(i)$. First, note that $v_{1}$ cannot be a 3 -vertex by Lemma 2|(iii). So both $v_{0}$ and $v_{2}$ are 3 -vertices. By the minimality of $G$, the graph $G-v v_{0}$ has a nice colouring, which we extend to a partial 10-edge-face colouring of $G$ in which only $v v_{0}$ and the $(\leqslant 4)$-faces are uncoloured. We obtain a contradiction by properly colouring $v v_{0}$, thereby exhibiting a nice colouring of $G$. If $v v_{0}$ cannot be coloured greedily, then we may assume without loss of generality that the colouring is the one shown in Figure $2(a)$.

First, observe that $\{\alpha, \beta, \gamma\}=\{8,9,10\}$, for otherwise we can recolour $v v_{2}$ with $x \in\{8,9,10\} \backslash\{\alpha, \beta, \gamma\}$ and then colour $v v_{0}$ with 2. Consequently, if $\delta \neq 1$ then we can interchange the colours of $v v_{1}$ and $v_{1} v_{2}$, i.e. recolour $v v_{1}$ with $\alpha$ and $v_{1} v_{2}$ with 1 . Now $v v_{0}$ can be properly coloured with 1 . Thus, $\delta=1$.

Since $\beta \neq \gamma$, there exists a colour $c \in\{\beta, \gamma\} \backslash\{9\}$. Note that $c \in\{8,10\}$ and $c \neq \alpha$. Hence, $c \in \mathcal{E}\left(v_{1}\right) \backslash\{1,9, \alpha\}$, for otherwise we recolour $v v_{1}$ with $c$ and then colour $v v_{0}$ with 1 to obtain a nice colouring of $G$. Similarly, if there is a colour $x \in\{2,3, \ldots, 7\} \backslash\left(\mathcal{E}\left(v_{1}\right) \cup\{\varepsilon\}\right)$, we recolour $v_{0} v_{1}$ with $x$ and colour $v v_{0}$ with 9. Thus, $\mathcal{E}\left(v_{1}\right) \cup\{\varepsilon\}=\{1,2, \ldots, 7,9, \alpha, c\}=\{1,2, \ldots, 10\}$. Since $\operatorname{deg}\left(v_{1}\right) \leqslant 9$, we deduce that $\varepsilon \notin \mathcal{E}\left(v_{1}\right)$. So, $\varepsilon \in\{2,3, \ldots, 7\}$ as $\varepsilon \notin\{8,9,10\}$ recalling that $G$ has no cut-edge by Lemma 2| $(i)$. We recolour $v_{1} v_{2}$ with $\varepsilon$ and $v v_{2}$ with $\alpha$. Now, colouring $v v_{0}$ with 2 yields a nice colouring of $G$; a contradiction.

(ii). Lemma 2( $\left(\right.$ iii) implies that none of $v_{1}, v_{2}$ and $v_{3}$ is a 3 -vertex. So both $v_{0}$ and $v_{4}$ are 3 -vertices. As in $(i)$, we obtain a partial 10-edge-face colouring of $G$ in which only $v v_{2}$ and the $(\leqslant 4)$-faces are uncoloured. Without loss of generality, we assume that the colouring is the one shown in Figure $2(b)$. Note that $\{8,9,10\} \subset \mathcal{E}\left(v_{2}\right)$, for otherwise we could greedily colour $v v_{2}$. Hence, at least one of $\{1,2\}$ and $\{3,4\}$ is disjoint from $\mathcal{E}\left(v_{2}\right)$. We may assume by symmetry that $\{1,2\} \cap \mathcal{E}\left(v_{2}\right)=\emptyset$. We can now proceed as in $(i)$. More precisely, we first note that $\{\alpha, \beta, \gamma\}=\{8,9,10\}$, since otherwise we could recolour $v v_{0}$ with 8,9 or 10 and then colour $v v_{2}$ with 1 . Moreover, if $\delta \neq 2$, then we can interchange the colours of $v v_{1}$ and $v_{0} v_{1}$ (i.e. recolour $v v_{1}$ with $\alpha$ and $v_{0} v_{1}$ with 2), and colour $v v_{2}$ with 2 . So $\delta=2$.

Now we observe that no edge incident to $v_{1}$ is coloured 1 . Indeed, since $\operatorname{deg}\left(v_{1}\right) \leqslant 9$, there is a colour $x$ not assigned to an edge incident to $v_{1}$. If $x \in\{8,9,10\}$, we recolour $v v_{1}$ with $x$ and then colour $v v_{2}$ with 2. If $x \in\{3,4, \ldots, 7\}$, we recolour $v_{0} v_{1}$ with $x, v v_{0}$ with $\alpha$ and then colour $v v_{2}$ with 1 . Therefore $x=1$ (since 2 is assigned to $v v_{1}$ ). As a result, we can safely 


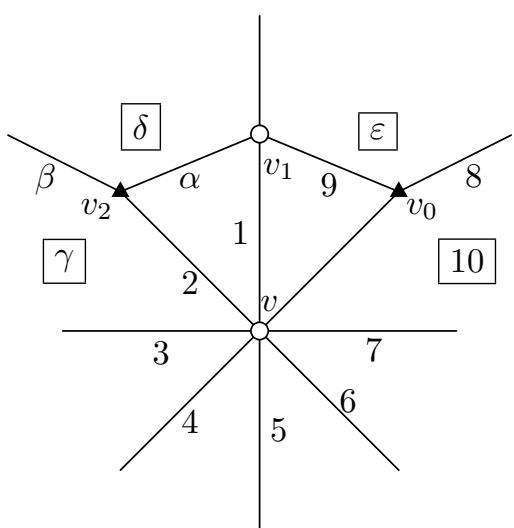

(a)

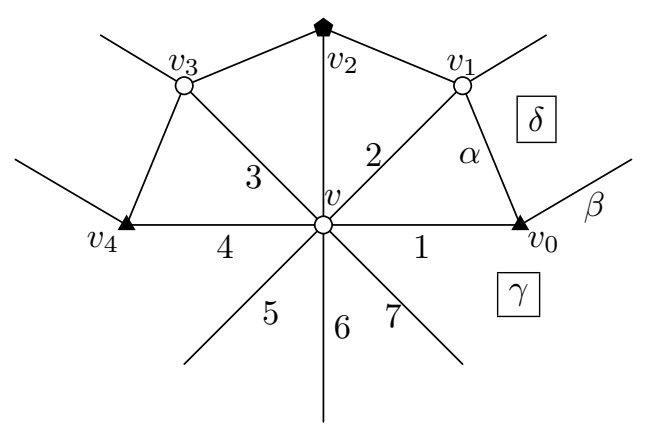

(b)

Figure 2: Reducible configurations for the proof of Lemma 5.

interchange the colours of $v v_{0}$ and $v_{0} v_{1}$, and then colour $v v_{2}$ with 1 .

Lemma 6. The graph $G$ satisfies the following assertions.

(i) The configuration of Figure $3(a)$ is reducible.

(ii) The configuration of Figure $3(b)$ is reducible.

(iii) The configuration of Figure $3(c)$ is reducible.

Proof. (i). Suppose on the contrary that $G$ contains the configuration of Figure 3( $a)$. By the minimality of $G$, the graph $G-v v_{8}$ has a nice colouring, from which we obtain a partial 10-edge-face colouring with only the edge $v v_{8}$ and the $(\leqslant 4)$-faces left uncoloured. It suffices to properly colour the edge $v v_{8}$ to obtain a nice colouring of $G$, which would lead to a contradiction. If the edge $v v_{8}$ cannot be coloured greedily, then $\left|\mathcal{C}\left(v v_{8}\right)\right|=10$, so we may assume the colouring is the one shown in Figure $3(a)$.

First, note that $\{9,10\}=\{\alpha, \beta\}$, for otherwise we could recolour $v v_{6}$ with 9 or 10 and then colour $v v_{8}$ with 7 . Similarly, $\beta \in \mathcal{E}\left(v_{7}\right)$, otherwise we recolour $v v_{7}$ with $\beta$ and colour $v v_{8}$ with 8 . Now $\gamma=8$, for otherwise we interchange the colours of $v_{6} v_{7}$ and $v v_{7}$ (i.e. we recolour $v_{6} v_{7}$ with 8 and $v v_{7}$ with $\alpha$ ) and colour $v v_{8}$ with 8 . Since $\operatorname{deg}\left(v_{7}\right) \leqslant 9$ and $\{8,9,10\}=\{8, \alpha, \beta\} \subset \mathcal{E}\left(v_{7}\right)$, there exists a colour $x \in\{1,2, \ldots, 7\} \backslash \mathcal{E}\left(v_{7}\right)$. As $\gamma=8$, we can recolour $v_{6} v_{7}$ with $x$ and $v v_{6}$ with $\alpha$, and colour $v v_{8}$ with 7 to obtain a nice colouring of $G$.

$(\mathrm{ii})$. Suppose on the contrary that $G$ contains the configuration of Figure $3(b)$. By the minimality of $G$, the graph $G-v v_{2}$ has a nice colouring, 
from which we obtain a partial 10-edge-face colouring with only $v v_{2}$ and the $(\leqslant 4)$-faces uncoloured. It suffices to properly colour the edge $v v_{2}$ to obtain a nice colouring of $G$, and therefore a contradiction. If the edge $v v_{2}$ cannot be coloured greedily, then $\left|\mathcal{C}\left(v v_{2}\right)\right|=10$ and thus we may assume that the colouring is the one shown in Figure $3(b)$.

First, note that $\{9,10\} \subseteq\{\alpha, \beta, \gamma\}$, for otherwise we could recolour $v v_{0}$ with 9 or 10 and then colour $v v_{2}$ with 7 . Furthermore, $10 \in \mathcal{E}\left(v_{1}\right)$, otherwise we recolour $v v_{1}$ with 10 and colour $v v_{2}$ with 8. If there exists a colour $x \in\{1,2, \ldots, 7\} \backslash\left(\{\varepsilon\} \cup \mathcal{E}\left(v_{1}\right)\right)$ then we recolour $v_{1} v_{2}$ with $x$ and colour $v v_{2}$ with 9. Hence, $\{1,2, \ldots, 10\}=\{\varepsilon\} \cup \mathcal{E}\left(v_{1}\right)$. Since $\left|\mathcal{E}\left(v_{1}\right)\right|=\operatorname{deg}\left(v_{1}\right) \leqslant 9$, we deduce that $\varepsilon \notin \mathcal{E}\left(v_{1}\right)$, and in particular $\varepsilon \neq 8$.

Suppose that $\alpha \neq 10$, and thus $\{\beta, \gamma\}=\{9,10\}$. We recolour $v_{1} v_{2}$ with 8 , $v v_{1}$ with $9, v v_{0}$ with 8 , and then colour $v v_{2}$ with 7 (note that $\alpha \neq 8$ ).

Hence, $\alpha=10$. So $8 \in\{\beta, \gamma\}$, otherwise we recolour $v v_{0}$ with $8, v v_{1}$ with $9, v_{1} v_{2}$ with 8 , and then colour $v v_{2}$ with 7 . Thus, $\{\beta, \gamma\}=\{8,9\}$, and consequently $\delta \notin\{8,9,10\}$. If $\beta=9$ we recolour $v_{0} v_{1}$ with $8, v v_{1}$ with 10 and then colour $v v_{2}$ with 8 . If $\beta=8$ we recolour $v_{0} v_{1}$ with $9, v_{1} v_{2}$ with $8, v v_{1}$ with 10 and then colour $v v_{2}$ with 9 .

(iii). Suppose on the contrary that $G$ contains the configuration of Figure $3(c)$. By the minimality of $G$, the graph $G-v v_{0}$ has a nice colouring, from which we infer a partial 10-edge-face colouring of $G$ in which only $v v_{0}$ and the $(\leqslant 4)$-faces are left uncoloured. We now obtain a nice colouring of $G$ by showing that the edge $v v_{0}$ can be properly coloured. If $v v_{0}$ cannot be coloured greedily, then $\left|\mathcal{C}\left(v v_{0}\right)\right|=10$ and, up to permuting the colours, we may assume that the colouring is the one shown in Figure $3(c)$.

First, note that $\{\alpha, \beta\}=\{9,10\}$, otherwise $v v_{6}$ can be recoloured with 9 or 10 and then $v v_{0}$ can be coloured with 6 . Similarly, $\beta \in \mathcal{E}\left(v_{7}\right)$ for otherwise we recolour $v v_{7}$ with $\beta$ and colour $v v_{0}$ with 7 . Moreover, if $\gamma \neq 7$ then we interchange the colours of $v v_{7}$ and $v_{6} v_{7}$ and colour $v v_{0}$ with 7 . Thus, $\gamma=7$.

Since $\operatorname{deg}\left(v_{7}\right) \leqslant 9$, there exists a colour $x \in\{1,2, \ldots, 10\} \backslash \mathcal{E}\left(v_{7}\right)$. Note that $x \notin\{7,9,10\}=\{7, \alpha, \beta\} \subset \mathcal{E}\left(v_{7}\right)$. Thus, since $\gamma=7$, we can recolour $v_{6} v_{7}$ with $x$ and $v v_{6}$ with $\alpha$, and colour $v v_{0}$ with 6 to obtain a nice colouring of $G$.

We end this section with a lemma that will help us deal with $(\geqslant 6)$-faces. An edge $u v$ is light if $\operatorname{deg}(u)+\operatorname{deg}(v) \leqslant 9$.

Lemma 7. Let $f$ be a $d$-face of $G$ for $d \geqslant 6$. Let $q$ be the number of 2-vertices and $\ell$ the number of light edges incident to $f$. If $\ell \geqslant 1$ then $q+\ell \leqslant 2 d-10$.

Proof. Suppose on the contrary that $\ell \geqslant 1$ and $q+\ell \geqslant 2 d-9$. Let $\mathscr{L}$ be the set of light edges incident to $f$. Since $|\mathscr{L}|=\ell \geqslant 1$, let $e_{0} \in \mathscr{L}$. By the 


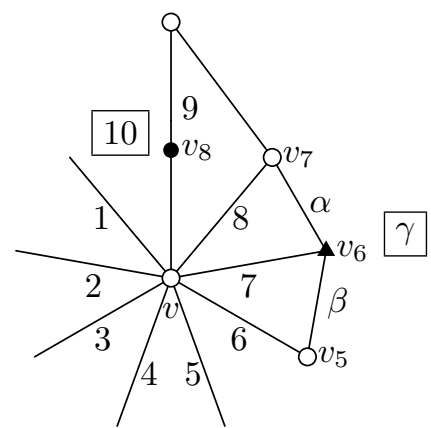

(a)

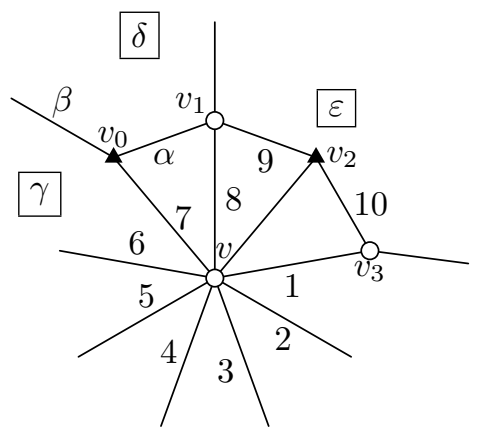

(b)

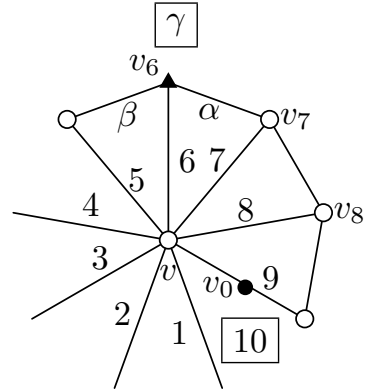

(c)

Figure 3: Reducible configurations of Lemma 6.

minimality of $G$, the graph $G-e_{0}$ has a nice colouring, from which we obtain a partial 10-edge-face colouring of $G$ in which only $e_{0}, f$ and the $(\leqslant 4)$-faces are uncoloured. We furthermore uncolour all the edges in $\mathscr{L}$. Now it suffices to properly colour the face $f$ and the edges in $\mathscr{L}$ to obtain a nice colouring of $G$, and hence a contradiction.

First, note that $f$ is adjacent to at most $d-q$ other faces. Furthermore, $f$ is incident to at most $d-\ell$ coloured edges. Hence, $|\mathcal{C}(f)| \leqslant 2 \cdot d-q-\ell \leqslant 9$. So we can greedily colour $f$.

It remains to colour the edges of $\mathscr{L}$. To this end, we build an auxiliary graph $H$ with vertex set $\mathscr{L}$, and for every pair $\left(e, e^{\prime}\right) \in \mathscr{L}^{2}$, we add an edge in $H$ between $e$ and $e^{\prime}$ if and only if $e$ and $e^{\prime}$ are adjacent in $G$. Recall that $\mathcal{F}(e)=\{1,2, \ldots, 10\} \backslash \mathcal{C}(e)$. Observe that properly colouring the edges of $G$ in $\mathscr{L}$ amounts to properly colouring the vertices of $H$ so that each $e \in \mathscr{L}$ is assigned a colour from $\mathcal{F}(e)$. Such a colouring of $H$ is an $\mathcal{F}$-colouring.

For each edge $e=u v \in \mathscr{L}$, note that $|\mathcal{C}(e)| \leqslant \operatorname{deg}(u)+\operatorname{deg}(v)-2+2-$ $\operatorname{deg}_{H}(e) \leqslant 9-\operatorname{deg}_{H}(e)$ since $e$ is light. Hence, $|\mathcal{F}(e)| \geqslant 1+\operatorname{deg}_{H}(e)$. As a result, we can (properly) greedily colour each vertex $e$ of $H$ with a colour from $\mathcal{F}(e)$. Indeed, given any partial colouring of $H$ and any $e \in \mathscr{L}$, the number of colours available to colour $e$ is at least $|\mathcal{F}(e)|-\operatorname{deg}_{H}(e) \geqslant 1$. This concludes the proof.

\section{Discharging part}

Recall that $G=(V, E)$ is a plane graph that is a minimum counter-example to the statement of Theorem 1 , in the sense that $|E|$ is minimum. (In particular, a planar embedding of $G$ is fixed.) We obtain a contradiction by using the Discharging Method. Here is an overview of the proof. Each vertex and face 
of $G$ is assigned an initial charge; the total sum of the charges is negative by Euler's Formula. Then vertices and faces send or receive charge according to certain redistribution rules. The total sum of the charges remains unchanged, but at the end we infer that the charge of each vertex and face is non-negative; a contradiction.

Initial charge. We assign a charge to each vertex and face. For every vertex $v \in V$, we define the initial charge $\operatorname{ch}(v)$ to be $2 \cdot \operatorname{deg}(v)-6$, while for every face $f \in F$, we define the initial charge $\operatorname{ch}(f)$ to be $\operatorname{deg}(f)-6$. The total sum is

$$
\sum_{v \in V} \operatorname{ch}(v)+\sum_{f \in F} \operatorname{ch}(f)=-12
$$

Indeed, by Euler's formula $|E|-|V|-|F|=-2$. Thus, $6|E|-6|V|-6|F|=$ -12. Since $\sum_{v \in V} \operatorname{deg}(v)=2|E|=\sum_{f \in F} \operatorname{deg}(f)$, it follows that

$$
\begin{aligned}
-12 & =4 \cdot|E|-6 \cdot|V|+\sum_{f \in F}(\operatorname{deg}(f)-6) \\
& =\sum_{v \in V}(2 \operatorname{deg}(v)-6)+\sum_{f \in F}(\operatorname{deg}(f)-6) .
\end{aligned}
$$

Rules. We need the following definitions to state the discharging rules. A 3 -face incident to a 3 -vertex is very-bad, and a 3 -face incident to a 4 - or 5 -vertex is bad. Furthermore, let $u$ be a 2 -vertex and $f$ a 4 -face incident to $u$. If $v$ is a neighbour of $u$ then $f$ is very-bad for $v$.

A face that is neither bad nor very-bad (for some vertex $v$ ) is safe (for $v$ ). Note that a very-bad 3-face cannot be bad by Lemma 2 2(iii). Recall that a 3 -face with vertices $x, y$ and $z$ is a $(\operatorname{deg}(x), \operatorname{deg}(y), \operatorname{deg}(z))$-triangle.

Recall that $G$ may have cut-vertices (of a type not forbidden by Lemma $2(i)$ ). Thus, in the rules below, when we say that a vertex $v$ sends charge to an incident face $f$, we mean that the charge is sent as many times as $v$ appears on the boundary walk of $f$. As we shall see, faces send charge only to 2-vertices, and a 2 -vertex cannot be incident twice to the same face (since $G$ has no cut-edge).

Rule R0. An ( $\geqslant 4)$-face sends 1 to every incident 2-vertex.

Rule R1. An ( $\geqslant 8)$-vertex sends $3 / 2$ to each of its incident very-bad faces; $5 / 4$ to each incident bad face; and 1 to each incident safe face.

Rule R2. A 7-vertex sends 8/7 to each incident face. 
Rule R3. A 6-vertex sends 1 to each incident face.

Rule R4. A 5-vertex sends $4 / 5$ to each incident face.

Rule R5. A 4-vertex sends $2 / 3$ to each incident $(4,7, \geqslant 8)$-triangle; and $1 / 2$ to each incident $(4, \geqslant 8, \geqslant 8)$-triangle.

In the sequel, we prove that the final charge $\operatorname{ch}^{*}(x)$ of every $x \in V \cup F$ is non-negative. Hence, we obtain

$$
-12=\sum_{x \in V \cup F} \operatorname{ch}(x)=\sum_{x \in V \cup F} \operatorname{ch}^{*}(x) \geqslant 0,
$$

a contradiction. This contradiction establishes the theorem.

Final charge of faces. Let $f$ be a $d$-face. Our goal is to show that $\operatorname{ch}^{*}(f) \geqslant 0$. Recall that the initial charge of $f$ is $\operatorname{ch}(f)=\operatorname{deg}(f)-6$.

We first focus on the case where $d \geqslant 6$. Let $v_{1}, v_{2}, \ldots, v_{d}$ be the vertices incident to $f$ in clockwise order. Let $p$ be the number of $(\geqslant 6)$-vertices incident to $f$, and $q$ the number of 2 -vertices incident to $f$. Lemma $3(i)$ implies that $q \leqslant\left\lfloor\frac{d}{2}\right\rfloor$. Hence, by Rules R0-R5, the final charge of $f$ is $\operatorname{ch}^{*}(f) \geqslant d-6+p-q$. In particular, $\operatorname{ch}^{*}(f) \geqslant 0$ provided $p-q \geqslant 6-d$. We now prove that $p-q \geqslant 6-d$ for all $d \geqslant 6$.

If $d \geqslant 11$ then $d-q \geqslant\left\lceil\frac{d}{2}\right\rceil \geqslant 6$ since $q \leqslant\left\lfloor\frac{d}{2}\right\rfloor$. Thus, $p-q \geqslant 6-d$, as wanted. We obtain the desired conclusion for the cases where $d \in\{6,7,8,9,10\}$ by applying Lemma 7. Note that $\operatorname{ch}^{*}(f) \geqslant 0$ if $q=0$ since $d \geqslant 6$. So we assume that $q \geqslant 1$. Let $\ell$ be the number of light edges of $f$. If $\ell=0$, then $p \geqslant q$ and hence $\operatorname{ch}^{*}(f) \geqslant 0$ since $d \geqslant 6$. So we assume that $\ell \geqslant 1$. Therefore Lemma 7 implies that $q+\ell \leqslant 2 d-10$. Observe that $\ell \geqslant 2(q-p)$. Hence, $2(q-p) \leqslant 2 d-10-q$. Consequently, if $q \geqslant 1$ then $2 d-10-q \leqslant 2 d-11$ and hence $q-p \leqslant d-6$ because $q-p$ is an integer.

Suppose now that $d=5$. By Lemma $2($ ii $)$, the face $f$ has no light edge. Hence, either $f$ is incident only to $(\geqslant 5)$-vertices, in which case $\operatorname{ch}^{*}(f) \geqslant$ $5-6+5 \cdot \frac{4}{5}=3>0$ by Rules R1-R4, or $f$ is incident to an $(\leqslant 4)$-vertex $v$, in which case the two neighbours of $v$ on $f$ are $(\geqslant 6)$-vertices by Lemma $2(i i)$ and therefore

$$
\operatorname{ch}^{*}(f) \geqslant \min (5-6-1 \cdot 1+2 \cdot 1,5-6-2 \cdot 1+3 \cdot 1)=0
$$

by Rules R0-R3.

We now suppose that $d=4$, i.e. $f$ is a 4 -face. If $f$ is not incident to a 2-vertex, then by Lemma 2|(iii) the face $f$ is incident to at least two $(\geqslant 6)$-vertices. Therefore its final charge is $\operatorname{ch}^{*}(f) \geqslant 4-6+2 \cdot 1=0$. 
If $f$ is incident to a 2-vertex, then $f$ is very-bad. By Lemma 3)( $($ iii $)$, all the other vertices incident to $f$ have degree at least 4 . Furthermore, Lemma 2( $($ iii $)$ implies that the face $f$ is incident to (at least) two 9-vertices $u$ and $v$, namely the two neighbours of the 2 -vertex. Thus, $f$ is very-bad for $u$ and for $v$. Therefore $f$ receives at least $2 \cdot \frac{3}{2}=3$ by Rule R1, and sends 1 to its incident 2-vertex by Rule R0. Consequently, the final charge of $f$ is $\operatorname{ch}^{*}(f) \geqslant 4-6+2 \cdot \frac{3}{2}-1=0$.

Finally, assume that $f$ is an $(x, y, z)$-triangle, with $x \leqslant y \leqslant z$. First, Lemma $3($ ii $)$ implies that $f$ is not incident to a 2 -vertex. Thus, Rule R0 does not apply to $f$, and therefore $f$ sends nothing. We consider several cases regarding the value of $x$.

$x \geqslant 6$. Then by Rules R1, R2 and R3, the final charge of $f$ is $\operatorname{ch}^{*}(f) \geqslant$ $3-6+3 \cdot 1=0$.

$x=5$. In this case, $f$ is bad. Moreover, Lemma 2( $($ iii $)$ implies that $y \geqslant 6$. If $y \geqslant 7$, then the final charge of $f$ is $\operatorname{ch}^{*}(f) \geqslant 3-6+\frac{4}{5}+2 \cdot \frac{8}{7}=\frac{3}{35}>0$, by Rules R1, R2 and R4. If $y=6$, then Lemma 4 implies that $z \geqslant 8$. Therefore by Rules R1, R3 and R4, the final charge of $f$ is $\operatorname{ch}^{*}(f)=3-6+\frac{4}{5}+1+\frac{5}{4}=\frac{1}{20}>0$.

$x=4$. Then $f$ is bad. It follows from Lemmas 2|(iii) and 4 that $y \geqslant 7$ and $z \geqslant 8$. If $y \geqslant 8$ then by Rules R 1 and R5 the final charge of $f$ is $\operatorname{ch}^{*}(f) \geqslant 3-6+\frac{1}{2}+2 \cdot \frac{5}{4}=0$. If $y=7$ then $f$ receives $2 / 3$ from its 4 -vertex by Rule R5. Furthermore, $f$ receives $8 / 7$ from its 7 -vertex by Rule R2, and $5 / 4$ from its $(\geqslant 8)$-vertex by Rule R1. Thus, its final charge is $\operatorname{ch}^{*}(f)=3-6+\frac{2}{3}+\frac{8}{7}+\frac{5}{4}=\frac{5}{84}>0$.

$x=3$. The face $f$ is very-bad, and it follows from Lemma 2N(iii) that $y \geqslant 8$. Therefore by Rule $\mathrm{R} 1$ the face $f$ receives $2 \cdot \frac{3}{2}=3$. Thus, its final charge is $\operatorname{ch}^{*}(f) \geqslant 3-6+2 \cdot \frac{3}{2}=0$.

Final charge of vertices. Let $v$ be an arbitrary vertex of $G$. Our goal is to show that $\operatorname{ch}^{*}(v) \geqslant 0$. Recall that the initial charge of $v$ is $\operatorname{ch}(v)=2 \cdot \operatorname{deg}(v)-6$. Moreover, $\operatorname{deg}(v) \geqslant 2$ by Lemma $2(i)$.

If $\operatorname{deg}(v)=2$, then $v$ is incident to two distinct $(\geqslant 4)$-faces by Lemmas $2 \|(i)$ and $3($ (ii) . Each of those two faces gives 1 to $v$ by Rule R0. Thus, the final charge of $v$ is $\operatorname{ch}^{*}(v)=-2+2=0$.

If $\operatorname{deg}(v)=3$, then $v$ neither sends nor receives any charge. Hence, the final charge of $v$ is $\operatorname{ch}^{*}(v)=\operatorname{ch}(v)=0$.

Suppose now that $\operatorname{deg}(v)=4$. If $v$ is not incident to a $(4,7, \geqslant 8)$-triangle then by Rule R5 the final charge of $v$ is $\operatorname{ch}^{*}(v) \geqslant 2-4 \cdot \frac{1}{2}=0$. If $v$ is incident 
to a $(4,7, \geqslant 8)$-triangle, then Lemma $2 \mid($ iii $)$ implies that the edge between $v$ and the 7 -vertex is incident to an $(\geqslant 5)$-face. Hence, $v$ is incident to at most three 3-faces, and therefore the final charge of $v$ is $\operatorname{ch}^{*}(v) \geqslant 2-3 \cdot \frac{2}{3}=0$.

Suppose that $\operatorname{deg}(v) \in\{5,6,7\}$. By Rules R2, R3 and R4 the vertex $v$ sends $\frac{\operatorname{ch}(v)}{\operatorname{deg}(v)}$ to each of its incident faces. Therefore the final charge of $v$ is $\operatorname{ch}^{*}(v)=0$.

Suppose that $\operatorname{deg}(v)=8$. By Lemma 2( $($ iii $)$, every very-bad face incident to $v$ is a $(3,8, \geqslant 8)$-triangle. Thus, Lemma $5(i)$ implies that $v$ is incident to at most four very-bad faces. Let $v_{0}, v_{1}, \ldots, v_{7}$ be the neighbours of $v$ in clockwise order, and for $i \in\{0,1, \ldots, 7\}$ let $f_{i}$ be the face incident to $v v_{i}$ and $v v_{i+1}$, where the indices are taken modulo 8 . For every $i \in\{0,1, \ldots, 7\}$, we observe that if $f_{i}, f_{i+1}, \ldots, f_{i+j}$ are all bad faces and $j \in\{2,4\}$, then Lemma 2(iii) implies that one of $v_{i}$ and $v_{i+j+1}$ is a 4 - or a 5 -vertex, where the indices are taken modulo 8. Furthermore, let us note that Lemma 2)(iii) also implies that every very-bad face is adjacent to a safe face. We consider several cases regarding the number $x$ of very-bad faces for $v$. Recall that $x \leqslant 4$.

$x=0$. Then the final charge of $v$ is $\operatorname{ch}^{*}(v) \geqslant 10-8 \cdot \frac{5}{4}=0$.

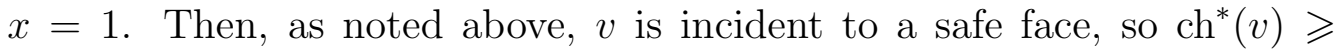
$10-\frac{3}{2}-6 \cdot \frac{5}{4}-1 \cdot 1=0$.

$x=2$. We assert that $v$ is incident to at least two safe faces. This yields the desired conclusion since it implies that $\operatorname{ch}^{*}(v) \geqslant 10-2 \cdot \frac{3}{2}-4 \cdot \frac{5}{4}-2 \cdot 1=0$. Without loss of generality, suppose that $f_{0}$ is very-bad. Since every very-bad face incident to $v$ is adjacent to a safe face, we can assume without loss of generality that $f_{7}$ is safe. Moreover, we also assume that $f_{6}$ is the second very-bad face, otherwise the assertion holds. Now, each of the faces $f_{1}, f_{2}, \ldots, f_{5}$ is either safe or bad. However, if all are bad faces, then one of $v_{1}$ and $v_{6}$ is a 4 - or a 5 -vertex, which is not possible since both $f_{0}$ and $f_{6}$ are $(3,8, \geqslant 8)$-triangles. So we deduce that $v$ is incident to at least two safe faces, as asserted.

$x=3$. We assert that $v$ is incident to at least three safe faces. This yields the desired conclusion since then $\operatorname{ch}^{*}(v) \geqslant 10-3 \cdot \frac{3}{2}-2 \cdot \frac{5}{4}-3 \cdot 1=0$. Since every very-bad face incident to $v$ is adjacent to a safe face, we infer the existence of an index $i$ such that $f_{i-1}$ and $f_{i+1}$ are very-bad, and $f_{i}$ is safe (for otherwise the conclusion holds), where the indices are taken modulo 8. Without loss of generality, we may assume that $f_{0}$ and $f_{6}$ are very-bad and $f_{7}$ is safe. Let $f_{j}$ be the third very-bad face incident to $v$. Then by symmetry $j \in\{1,2,3\}$. First, $j \neq 1$ by Lemma $5(i)$. If $j=2$, then necessarily $f_{1}$ is safe for $v$ by Lemma 2)( $(i i i)$. 
Furthermore, at least one face among $f_{3}, f_{4}, f_{5}$ is safe: none of these faces is very-bad and if they were all bad then one of $v_{3}$ and $v_{6}$ would be a 4 - or a 5 -vertex, which is not possible since both $f_{2}$ and $f_{6}$ are $(3,8, \geqslant 8)$-triangles. Finally, assume that $j=3$. Then at least one face among $f_{1}$ and $f_{2}$ is safe by Lemmas 2 $2(i i i)$ and $5($ ii $)$. Similarly, at least one face among $f_{4}$ and $f_{5}$ is safe, so $v$ is incident to three safe faces, as asserted.

$x=4$. Lemmas $2($ iii $)$ and $5(i)$ imply that $v$ is incident to four safe faces, so $\operatorname{ch}^{*}(v) \geqslant 10-4 \cdot \frac{3}{2}-4 \cdot 1=0$.

Finally, assume that $\operatorname{deg}(v)=9$. Let $v_{0}, v_{1}, \ldots, v_{8}$ be the neighbours of $v$ in clockwise order, and for $i \in\{0,1, \ldots, 8\}$ let $f_{i}$ be the face incident to $v v_{i}$ and $v v_{i+1}$, where the indices are taken modulo 9 .

Lemmas 2|(iii), 3(iii), G(i) and $6($ (ii) , imply that $v$ is not incident to three consecutive very-bad faces, i.e. there is no index $i$ such that all of $f_{i-1}, f_{i}$ and $f_{i+1}$ are very-bad (where the indices are taken modulo 9 ). To see this, suppose that $f_{0}, f_{1}$ and $f_{2}$ are all very-bad for $v$. The face $f_{1}$ cannot be a 4 -face, for otherwise one of $v_{1}$ and $v_{2}$ would be a 2-vertex, and so one of $f_{0}$ and $f_{2}$ would be safe for $v$ by Lemma 2|(iii). Hence, $f_{1}$ is a very-bad triangle and thus $v_{1}$ or $v_{2}$ is a 3 -vertex. By symmetry, we may assume that $v_{2}$ is a 3 -vertex, which implies that $v_{1}$ is an $(\geqslant 8)$-vertex by Lemma 2(iii). Consequently, we infer from Lemma $3($ iii $)$ that $f_{2}$ is not a very-bad 4 -face for $v$. So, $f_{2}$ is a very-bad triangle. Now, $f_{0}$ can neither be a very-bad triangle by Lemma $6($ ii $)$, nor a very-bad 4 -face for $v$ by Lemma $6(i)$; a contradiction. As a result, the number $x$ of very-bad faces for $v$ is at most 6 .

First, assume that $v$ is not incident to two consecutive very-bad faces. Thus, $x \leqslant 4$. We consider two cases depending on the value of $x$.

$x \leqslant 3$. Then the final charge of $v$ is $\operatorname{ch}^{*}(v) \geqslant 12-3 \cdot \frac{3}{2}-6 \cdot \frac{5}{4}=0$.

$x=4$. Without loss of generality, the very-bad faces incident to $v$ are $f_{0}, f_{2}, f_{4}$ and $f_{6}$. If $v$ is incident to at least one very-bad 4-face, then it is incident to at least one safe face by Lemma 2](iii), and the final charge of $v$ is $\operatorname{ch}^{*}(v) \geqslant 12-4 \cdot \frac{3}{2}-4 \cdot \frac{5}{4}-1=0$. So we may assume all the very-bad faces incident to $v$ are triangles. Consequently, $f_{1}, f_{3}$ and $f_{5}$ are safe by Lemma 2)( $(i i i)$. Therefore the final charge of $v$ is $\operatorname{ch}^{*}(v) \geqslant 12-4 \cdot \frac{3}{2}-2 \cdot \frac{5}{4}-3=\frac{1}{2}>0$.

It remains to deal with the case where $v$ is incident to two consecutive very-bad faces.

First, let us suppose that $v$ is incident to two consecutive very-bad faces one of which is a 4 -face. Without loss of generality, assume that $f_{0}$ and $f_{1}$ 
are very-bad for $v$, and $f_{0}$ is a 4 -face. Since $f_{1}$ is very-bad for $v$, we deduce from Lemma 2( $($ iii $)$ that $v_{0}$ is a 2-vertex. So $f_{8}$ is safe for $v$ by Lemma 2N(iii), and $v_{1}$ is an $(\geqslant 4)$-vertex by Lemma $3($ iii $)$. Moreover, $f_{2}$ is also safe for $v$ by Lemmas $2($ iii $)$ and $6(i)$. Thus, if $x \leqslant 5$ then $\operatorname{ch}^{*}(v) \geqslant 12-5 \cdot \frac{3}{2}-2 \cdot \frac{5}{4}-2=0$. If $x=6$ then, since $v$ is not incident to three consecutive very-bad faces, we deduce that $f_{3}, f_{4}, f_{6}$ and $f_{7}$ are very-bad for $v$. We prove that $f_{5}$ is then safe for $v$. This would yield that $\operatorname{ch}^{*}(v)=12-6 \cdot \frac{3}{2}-3 \cdot 1=0$, as wanted. If at least one of $f_{3}$ and $f_{4}$ is a 4 -face, then we infer as above that $f_{5}$ is safe for $v$ (and so is $f_{2}$ ). The same holds if one of $f_{6}$ and $f_{7}$ is a 4 -face. So we may assume that $f_{3}, f_{4}, f_{6}$ and $f_{7}$ are all triangles. Consequently, Lemma 2](iii) ensures that $f_{5}$ is safe. This concludes our analysis in this case since $x \leqslant 6$, as noted earlier.

Finally, assume that $v$ is not incident to two consecutive very-bad faces one of which is a 4 -face, but $v$ is incident to two consecutive very-bad faces. Without loss of generality, assume that $f_{0}$ and $f_{1}$ are two very-bad triangles. We consider several cases depending on the value of $x$. Recall that $x \leqslant 6$ since there are no three consecutive very-bad faces for $v$.

$x \leqslant 3$. Then the final charge of $v$ is $\operatorname{ch}^{*}(v) \geqslant 12-3 \cdot \frac{3}{2}-6 \cdot \frac{5}{4}=0$.

$x=4$. We assert that $v$ is incident to a safe face. This yields the result since then the final charge of $v$ is $\operatorname{ch}^{*}(v) \geqslant 12-4 \cdot \frac{3}{2}-4 \cdot \frac{5}{4}-1=0$. It remains to prove the assertion. Suppose on the contrary that $v$ is not incident to a safe face. Then all faces incident to $v$ are triangles (since every very-bad 4-face for $v$ is adjacent to a face that is safe for $v$ by Lemma 2(iii)). As a result, Lemma $6($ ii $)$ implies that $\operatorname{deg}\left(v_{1}\right)=3$. By Lemma 2| (iii), both $v_{0}$ and $v_{2}$ are $(\geqslant 8)$-vertices. Since $f_{2}=v v_{2} v_{3}$ and $f_{8}=v v_{0} v_{8}$ are bad faces, both $v_{3}$ and $v_{8}$ are $(\leqslant 5)$-vertices. Lemma $2(i i i)$ then implies that $v_{4}$ and $v_{7}$ are $(\geqslant 7)$-vertices. Since $x=4$, one of $v_{5}$ and $v_{6}$ is a 3 -vertex; by symmetry we may assume it is $v_{5}$. But now $\operatorname{deg}\left(v_{6}\right) \geqslant 8$ by Lemma 2](iii), so $v v_{6} v_{7}$ is a safe face; a contradiction.

$x=5$. We assert that $v$ is incident to at least two safe faces. This yields the conclusion since then $\operatorname{ch}^{*}(v) \geqslant 12-5 \cdot \frac{3}{2}-2 \cdot \frac{5}{4}-2=0$. Note that each of $f_{2}$ and $f_{8}$ is either bad or safe for $v$, since there are no three consecutive very-bad faces for $v$. Suppose first that none of $f_{2}$ and $f_{3}$ is safe for $v$. In particular, $f_{2}$ is bad. It then follows from Lemma 2](iii) that $v_{1}$ is a 3 -vertex and $v_{3}$ an $(\leqslant 5)$-vertex. Consequently, $f_{3}$ cannot be a very-bad triangle. Moreover, $f_{3}$ is not a very-bad 4 -face for $v$ by Lemma 6 ( $($ iii) $)$. So $f_{3}$ is a bad face. Now, three faces among $f_{4}, f_{5}, f_{6}$ and $f_{7}$ are very-bad for $v$, since $f_{8}$ cannot be very-bad for $v$. If both $f_{4}$ and $f_{5}$ are very-bad for $v$, then both are triangles and $f_{7}$ is also very-bad. 
Since $f_{3}$ is bad, we deduce that $v_{5}$ is a 3 -vertex. Consequently $f_{7}$ must be a 4 -face, for otherwise both $f_{6}$ and $f_{8}$ are safe. Thus, one of $f_{6}, f_{8}$ is safe. Further, the other face cannot be bad either by Lemma 6I(iii), and hence it is also safe for $v$. Consequently, the assertion holds if none of $f_{2}$ and $f_{3}$ is safe for $v$. By symmetry, the same argument applies to $f_{7}$ and $f_{8}$, and thus $v$ is incident to at least two safe faces, as asserted.

$x=6$. Then the very-bad faces are $f_{0}, f_{1}, f_{3}, f_{4}, f_{6}$ and $f_{7}$. Hence, they are all very-bad triangles. Consequently, $f_{2}, f_{5}$ and $f_{8}$ are safe for $v$ by Lemma $2($ iii $)$, Hence, the final charge of $v$ is $\operatorname{ch}^{*}(v)=12-6 \cdot \frac{3}{2}-3=0$.

This establishes that the final charge of every vertex is non-negative, so the proof of Theorem 1 is now complete.

Acknowledgement. The authors warmly thank Ross Kang and the referees for their careful reading of the paper. The authors learned that Lisa Macon independently proved the same result (i.e. Theorem 1) in her Ph.D. thesis.

\section{References}

[1] M. Behzad. Graphs and their chromatic numbers. Doctoral thesis, Michigan State University, 1965.

[2] O. V. Borodin. Coupled colorings of graphs on a plane (in Russian). Metody Diskret. Anal., 45:21-27, 95, 1987.

[3] O. V. Borodin. On the total coloring of planar graphs. J. Reine Angew. Math., 394:180-185, 1989.

[4] O. V. Borodin. Simultaneous coloring of edges and faces of plane graphs. Discrete Math., 128(1-3):21-33, 1994.

[5] O. V. Borodin, A. V. Kostochka, and D. R. Woodall. List edge and list total colourings of multigraphs. J. Combin. Theory Ser. B, 71(2):184-204, 1997.

[6] O. V. Borodin, A. V. Kostochka, and D. R. Woodall. Total colorings of planar graphs with large maximum degree. J. Graph Theory, 26(1):53-59, 1997.

[7] J. Fiamčík. Simultaneous colouring of 4-valent maps. Mat. Časopis Sloven. Akad. Vied, 21:9-13, 1971. 
[8] E. Jucovič. On a problem in map coloring. Mat. Časopis Sloven. Akad. Vied, 19:225-227, 1969. Errata ibid. 20:224, 1970.

[9] R. J. Kang, J.-S. Sereni, and M. Stehlík. Every plane graph of maximum degree 8 has an edge-face 9-colouring. Submitted for publication, 2009.

[10] L. Kowalik, J.-S. Sereni, and R. Škrekovski. Total-coloring of plane graphs with maximum degree nine. SIAM J. Discrete Math., 22(4):1462-1479, 2008 .

[11] L. S. Mel'nikov. Problem 9. In M. Fiedler, editor, Recent Advances in Graph Theory, Proc. International Symposium, Prague, 1974, page 543. Academia Praha, 1975.

[12] D. P. Sanders and Y. Zhao. On simultaneous edge-face colorings of plane graphs. Combinatorica, 17(3):441-445, 1997.

[13] D. P. Sanders and Y. Zhao. On improving the edge-face coloring theorem. Graphs Combin., 17(2):329-341, 2001.

[14] V. G. Vizing. Some unsolved problems in graph theory (in Russian). Uspehi Mat. Nauk, 23(6 (144)):117-134, 1968.

[15] A. O. Waller. Simultaneously coloring the edges and faces of plane graphs. J. Combin. Theory Ser. B, 69(2):219-221, 1997.

[16] W. Wang. Total chromatic number of planar graphs with maximum degree ten. J. Graph Theory, 54(2):91-102, 2007. 